\title{
Effect of postoperative chemotherapy on blood glucose and lipid metabolism in patients with invasive breast cancer
}

\author{
Aiying Qi ${ }^{\#}$, Yanping Li $^{\#}$, Susu Yan, Huiying Sun, Meiling Zhao, Yuhui Chen \\ Department of General Surgery, the First Medical Center of the Chinese People's Liberation Army (PLA) General Hospital, Beijing, China \\ Contributions: (I) Conception and design: A Qi, Y Chen; (II) Administrative support: A Qi, Y Chen; (III) Provision of study materials or patients: All \\ authors; (IV) Collection and assembly of data: All authors; (V) Data analysis and interpretation: All authors; (VI) Manuscript writing: All authors; \\ (VII) Final approval of manuscript: All authors. \\ \#These authors contributed equally to this work and are co-first authors. \\ Correspondence to: Yuhui Chen. Attending Physician, Department of General Surgery, the First Medical Center of the Chinese PLA General Hospital, \\ 28 Fuxing Road, Haidian District, Beijing, China. Email: cyhnju@sina.com.
}

Backgroundi Chemotherapy can lead to abnormal metabolism and affect the quality of life of patients after
operation. Here we explore the effect of postoperative chemotherapy on blood glucose and lipid metabolism
in patients with invasive breast cancer and thus provide evidence for the prevention and treatment of blood
glucose and lipid disorders after surgery. Methods: From January 2019 to December 2020, data from 141 patients with invasive breast cancer in our hospital were retrospectively collected. The levels of fasting blood glucose and blood lipid profiles [including total cholesterol, triglyceride, high-density lipoprotein (HDL), and low-density lipoprotein (LDL)] were compared before and after chemotherapy. Meanwhile, the metabolic risk factors for abnormal blood glucose and lipid profiles were analyzed.

Results: Fasting blood glucose levels significantly increased after treatment $(5.21 \pm 0.89 \mathrm{vs} .4 .87 \pm 0.71 \mathrm{mmol} / \mathrm{L}$, $\mathrm{P}=0.000)$, as did those of triglyceride $(1.81 \pm 1.02$ vs. $1.26 \pm 0.67 \mathrm{mmol} / \mathrm{L}, \mathrm{P}=0.000)$, while HDL significantly decreased $(1.11 \pm 0.29$ vs. $1.32 \pm 0.33 \mathrm{mmol} / \mathrm{L}, \mathrm{P}=0.000)$. There were no significant differences in the levels of total cholesterol and LDL before and after treatment $(\mathrm{P}>0.05)$. Multivariate logistic regression analysis showed that anthracycline-based chemotherapy was a protective factor for elevated fasting blood glucose $[\mathrm{P}=0.035$, 95\% CI: $0.248(0.068-0.908)]$, whereas receiving $>6$ cycles of chemotherapy was a risk factor for elevated fasting blood glucose ( $\mathrm{P}=0.026,95 \%$ CI: 4.036 (1.178-13.825)].

Conclusions: Postoperative chemotherapy can lead to the elevated triglyceride and fasting blood glucose and decreased HDL in patients with breast cancer. Anthracycline-based chemotherapy is a protective factor for the increase of fasting blood glucose, and more than 6 cycles of chemotherapy is a risk factor for the increase of fasting blood glucose.

Keywords: Postoperative chemotherapy; invasive breast cancer; blood glucose; blood lipids

Submitted Jan 08, 2021. Accepted for publication Mar 29, 2021.

doi: 10.21037 /gs-21-141

View this article at: http://dx.doi.org/10.21037/gs-21-141

\section{Introduction}

Breast cancer is the most common malignancy in women, and its prevalence has continued to rise (1-3). With the advances in medical technology, the postoperative recurrence and mortality in women with breast cancer have been significantly reduced $(4,5)$, and most patients can survive for longer periods of time, with the condition being akin to a chronic disease. Thus, the quality of life after breast cancer surgery has increasingly become a concern. To lower the risk of breast cancer recurrence after surgery, postoperative chemotherapy is required for (I) patients with invasive tumors larger than $2 \mathrm{~cm}$, (II) lymph node- 
positive patients, (III) hormone receptor-negative patients, or (IV) human epidermal growth factor receptor 2 (HER2)positive patients. However, while killing residual tumor cells, postoperative chemotherapy can also lead to a variety of complications. Previous studies had confirmed that the characteristics of blood lipid metabolism were different between invasive breast cancer patients and benign breast tumor patients. Moreover, patients with different molecular types could have different characteristics of blood lipid metabolism. Moreover, recent studies have shown that neoadjuvant chemotherapy can cause abnormal glucose and lipid metabolism in breast cancer patients $(6,7)$. Therefore, we hypothesized that postoperative chemotherapy on blood glucose and lipid metabolism could also affect the metabolism of glucose and lipid in invasive breast cancer patients. However, the relevant data are still insufficient, and therefore we designed and performed the current study to clarify this issue. We present the following article in accordance with the STROBE reporting checklist (available at http://dx.doi.org/10.21037/gs-21-141).

\section{Methods}

\section{General data}

The clinical data of 141 invasive breast cancer patients who were treated in our center from January 2019 to December 2020 were retrospectively collected. The inclusion criteria were as follows: (I) with invasive breast cancer; (II) aged 1875 years; (III) receiving surgical treatment and subsequent chemotherapy in our hospital; and (IV) with complete clinicopathologic data. The exclusion criteria included the following: (I) not undergoing standardized chemotherapy as required; (II) with abnormal blood glucose and lipid metabolism; (III) with hepatic and renal dysfunction; (IV) with cardiac, cerebral, pulmonary, and/or other vital organ dysfunction; (V) males with breast cancer; (VI) with stage IV breast cancer at first presentation; (VII) with metastatic or recurrent breast cancer; (VIII) with thyroid disease; (IX) with accompanying malignant tumors; (X) pregnant or lactating women; and (XI) with special types of invasive breast cancer. A total of 141 patients with invasive breast cancer treated with postoperative chemotherapy were included during the study period according to the inclusion and exclusion criteria. Individual consent for this retrospective analysis was waived. The ethical approval statement was not required due to the following reasons: (I) the study protocol was consistent with the Declaration of Helsinki (as revised in 2013); (II) our study was a retrospectively observational study, and we only studied the clinical data of the patients, which would not bring any harm to the patients; and (III) the personal information provided by patients would not be disclosed.

\section{Treatment regimens}

At least 4 cycles of anthracycline-based chemotherapy regimen or non-anthracycline-based chemotherapy regimen were administered after surgery. At least 1 breast ultrasound was performed every 2 cycles. Targeted therapy was used in human epidermal growth factor receptor 2 (HER2)-positive patients, and radiotherapy was offered to patients in the presence of the following: (I) primary tumor with maximum diameter $\geq 5 \mathrm{~cm}$, or tumor invasion of breast skin or chest wall; (II) $\geq 4$ positive axillary lymph nodes; (III) breast-conserving surgery; (IV) stage $\mathrm{T}_{1-2}$ with 1-3 positive lymph nodes; (V) simple mastectomy combined with sentinel lymph node biopsy for stage $T_{1-2}$ breast cancer, with positive sentinel lymph nodes and without subsequent axillary lymph node dissection.

\section{Main measures}

The main indicators included the following: (I) fasting blood glucose; and (II) blood lipids status, including that of total cholesterol, triglycerides, high-density lipoprotein (HDL), and low-density lipoprotein (LDL). Other indicators included age, body mass index, involved site(s), history of diabetes, history of hypertension, and size of primary mass; estrogen receptor (ER), progesterone receptor (PR), Ki-67, HER2, and lymph node metastasis status; type of surgical procedure; total chemotherapy cycles; and the status of axillary lymph node dissection, postoperative endocrine therapy, radiotherapy, and chemotherapy regimen.

\section{Definitions}

The positive cutoffs for ER, PR, and Ki-67(\%) were $\geq 10 \%$, $\geq 10 \%$, and $\geq 14 \%$, respectively. Increased triglycerides or fasting glucose was defined as triglycerides or fasting glucose being higher after chemotherapy than before chemotherapy, while decreased HDL was defined as HDL being lower after chemotherapy than before chemotherapy.

\section{Quality control}

In our study, we designed strict inclusion and exclusion 
Table 1 Clinical features of 141 patients with invasive breast cancer treated with postoperative chemotherapy

\begin{tabular}{|c|c|}
\hline Clinical features & Values \\
\hline Age (years) & $49.13 \pm 9.65$ \\
\hline Body mass index $\left(\mathrm{kg} / \mathrm{m}^{2}\right)$ & $24.58 \pm 3.14$ \\
\hline \multicolumn{2}{|l|}{ Mass size before surgery } \\
\hline Length (cm) & $2.73 \pm 1.52$ \\
\hline Width (cm) & $2.07 \pm 1.21$ \\
\hline Height (cm) & $1.58 \pm 0.92$ \\
\hline Total chemotherapy cycles & $6.94 \pm 1.48$ \\
\hline \multicolumn{2}{|l|}{ Chemotherapy regimen } \\
\hline $\begin{array}{l}\text { Anthracycline-based chemotherapy } \\
\text { regimen }\end{array}$ & $99(70.21 \%)$ \\
\hline $\begin{array}{l}\text { Non-anthracycline-based chemotherapy } \\
\text { regimen }\end{array}$ & $42(29.79 \%)$ \\
\hline \multicolumn{2}{|l|}{ Site of breast cancer } \\
\hline Left & $76(53.90 \%)$ \\
\hline Right & $65(46.10 \%)$ \\
\hline History of diabetes & 7 (4.96\%) \\
\hline History of hypertension & $20(14.18 \%)$ \\
\hline ER positive & $89(63.12 \%)$ \\
\hline PR positive & $85(60.28 \%)$ \\
\hline Ki-67 positive & $128(90.78 \%)$ \\
\hline \multicolumn{2}{|l|}{ HER2 } \\
\hline$(-)$ & $20(14.18 \%)$ \\
\hline$(1+)$ & $30(21.28 \%)$ \\
\hline$(2+)$ & $60(42.55 \%)$ \\
\hline$(3+)$ & $31(21.99 \%)$ \\
\hline Lymph node positive & $80(56.74 \%)$ \\
\hline \multicolumn{2}{|l|}{ Surgical procedures } \\
\hline Total mastectomy & $128(90.78 \%)$ \\
\hline Breast-conserving surgery & $13(9.22 \%)$ \\
\hline Axillary lymph node dissection & $119(84.40 \%)$ \\
\hline Endocrine therapy & $94(66.67 \%)$ \\
\hline Radiotherapy & $92(65.25 \%)$ \\
\hline Targeted therapy & $40(28.37 \%)$ \\
\hline
\end{tabular}

criteria. In addition, we chose objective indicators to avoid recall bias. Finally, we chose two persons to input data independently. If the data was inconsistent, a third person would check it.

\section{Statistical analysis}

Statistical analysis was performed in the SPSS 22.0 software package (IBM Corp.), and a $\mathrm{P}$ value of less than 0.05 was considered statistically significant. Changes in glucose and lipids before and after chemotherapy were analyzed using paired samples $t$-test, and the data are presented with mean \pm standard deviation. Risk factors for changes in glucose and lipids in patients with invasive breast cancer were analyzed using multivariate logistic regression analysis.

\section{Results}

\section{Clinical features of 141 patients with invasive breast cancer treated with postoperative chemotherapy}

The clinical features of 141 patients with invasive breast cancer are summarized in Table 1.

\section{Comparison of glucose and lipids before and after chemotherapy}

Fasting blood glucose levels significantly increased after treatment $(5.21 \pm 0.89$ vs. $4.87 \pm 0.71 \mathrm{mmol} / \mathrm{L}, \mathrm{P}=0.000)$ as $\mathrm{did}$ triglyceride levels $(1.81 \pm 1.02$ vs. $1.26 \pm 0.67 \mathrm{mmol} / \mathrm{L}, \mathrm{P}=0.000)$, while those of HDL significantly decreased $(1.11 \pm 0.29 v s$. $1.32 \pm 0.33 \mathrm{mmol} / \mathrm{L}, \mathrm{P}=0.000)$. There were no significant differences in the levels of total cholesterol and LDL before and after treatment $(\mathrm{P}>0.05$; Table 2 and Figure 1$)$.

Factors influencing blood glucose in patients with invasive breast cancer treated with postoperative chemotherapy

Multivariate logistic regression analysis showed that anthracycline-based chemotherapy was a protective factor for elevated fasting blood glucose [ $\mathrm{P}=0.035,95 \%$ CI: 0.248 (0.068-0.908)], whereas receiving $>6$ cycles of chemotherapy was a risk factor for elevated fasting blood glucose $[\mathrm{P}=0.026$, 95\% CI: 4.036 (1.178-13.825); Table 3]. 
Table 2 Comparison of glucose and lipids before and after chemotherapy $(\mathrm{mmol} / \mathrm{L})$

\begin{tabular}{|c|c|c|c|c|c|}
\hline Categories & Fasting blood sugar & $\begin{array}{c}\text { Total cholesterol } \\
\text { (TC) }\end{array}$ & Triglyceride & $\begin{array}{l}\text { Low-density } \\
\text { lipoprotein }\end{array}$ & $\begin{array}{c}\text { High-density } \\
\text { lipoprotein }\end{array}$ \\
\hline Before chemotherapy $(n=141)$ & $4.87 \pm 0.71$ & $4.40 \pm 0.88$ & $1.26 \pm 0.67$ & $2.89 \pm 0.80$ & $1.32 \pm 0.33$ \\
\hline $\mathrm{t}$ & 5.427 & 0.915 & 8.478 & 0.591 & 9.539 \\
\hline
\end{tabular}

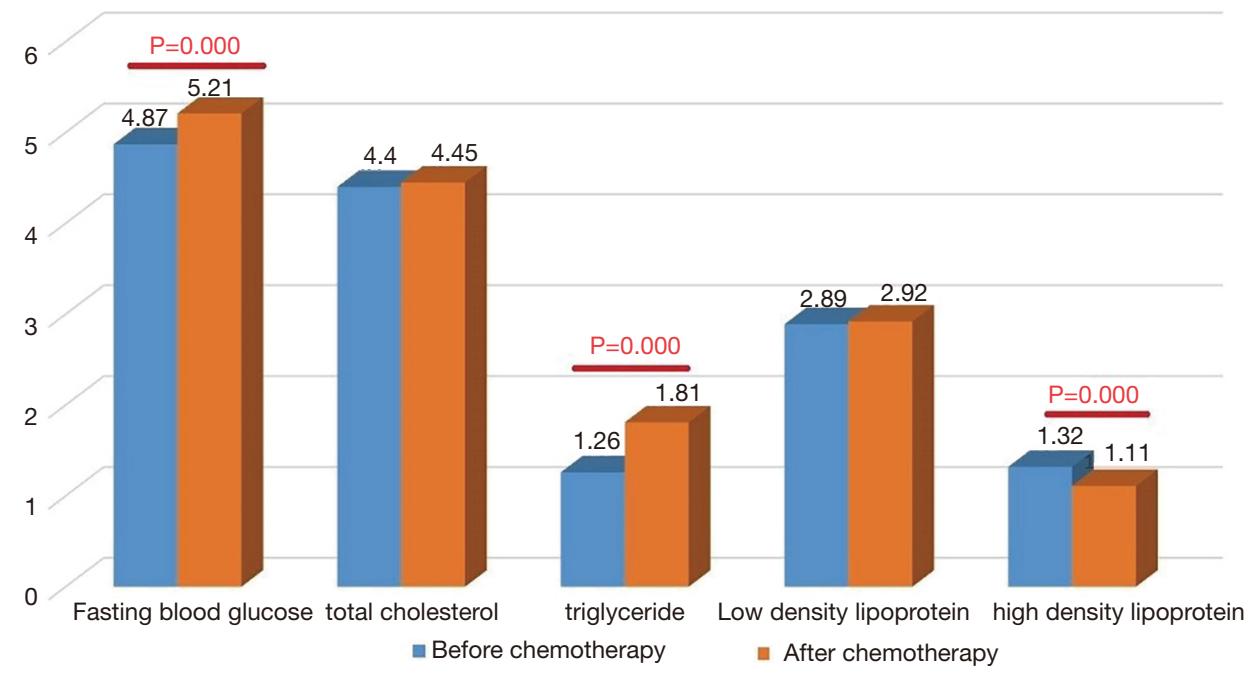

Figure 1 Comparison of glucose and lipids before and after chemotherapy (mmol/L).

Table 3 Factors influencing blood glucose in patients with invasive breast cancer treated with postoperative chemotherapy

\begin{tabular}{lccccc}
\hline Factors & B value & Standard error & Wald value & P value & 95\% confidence interval \\
\hline Constant coefficient & 0.885 & 0.530 & 2.788 & 0.095 & - \\
Endocrine therapy & -0.248 & 0.488 & 0.258 & 0.612 & $0.781(0.300-2.031)$ \\
Radiotherapy & 0.380 & 0.456 & 0.695 & 0.404 & $1.463(0.598-3.578)$ \\
Targeted therapy & 0.594 & 0.480 & 1.534 & 0.216 & $1.811(0.707-4.636)$ \\
Anthracycline-based chemotherapy regimen & -1.394 & 0.662 & 4.435 & 0.035 & $0.248(0.068-0.908)$ \\
$>6$ chemotherapy cycles & 1.395 & 0.628 & 4.932 & 0.026 & $4.036(1.178-13.825)$ \\
\hline
\end{tabular}

\section{Factors influencing increased triglyceride in patients with invasive breast cancer treated with postoperative chemotherapy}

The elevated triglycerides in patients with invasive breast cancer treated with postoperative chemotherapy was not significantly correlated with endocrine therapy, radiotherapy, targeted therapy, chemotherapy regimen, or chemotherapy cycle (all $\mathrm{P}>0.05 ;$ Table 4).

\section{Factors infuencing reduction of $H D L$ in patients with invasive breast cancer treated with postoperative chemotherapy}

The decreased HDL in patients with invasive breast cancer treated with postoperative chemotherapy was 
Table 4 Factors influencing increased triglyceride in patients with invasive breast cancer treated with postoperative chemotherapy

\begin{tabular}{lccccc}
\hline Factors & B value & Standard error & Wald value & P value & 95\% confidence interval \\
\hline Constant coefficient & 0.854 & 0.541 & 2.493 & 0.114 & - \\
Endocrine therapy & 0.063 & 0.512 & 0.015 & 0.903 & $1.065(0.390-2.903)$ \\
Radiotherapy & 0.207 & 0.494 & 0.176 & 0.675 & $1.230(0.467-3.240)$ \\
Targeted therapy & 0.515 & 0.527 & 0.954 & 0.329 & $1.673(0.595-4.702)$ \\
Anthracycline-based chemotherapy regimen & 0.767 & 0.788 & 0.948 & 0.330 & $2.154(0.460-10.089)$ \\
$>6$ chemotherapy cycles & -0.469 & 0.793 & 0.349 & 0.554 & $0.626(0.132-2.961)$ \\
\hline
\end{tabular}

Table 5 Factors influencing reduction of HDL in patients with invasive breast cancer treated with postoperative chemotherapy

\begin{tabular}{lccccc}
\hline Factors & B value & Standard error & Wald value & P value & 95\% confidence interval \\
\hline Constant coefficient & 0.936 & 0.569 & 2.708 & 0.100 & - \\
Endocrine therapy & 0.017 & 0.528 & 0.001 & 0.975 & $1.017(0.361-2.863)$ \\
Radiotherapy & 0.398 & 0.496 & 0.642 & 0.423 & $1.488(0.563-3.937)$ \\
Targeted therapy & 1.005 & 0.598 & 2.825 & 0.093 & $2.732(0.846-8.829)$ \\
Anthracycline-based chemotherapy regimen & 0.101 & 0.752 & 0.018 & 0.893 & $1.106(0.253-4.830)$ \\
$>$ 6 chemotherapy cycles & -0.089 & 0.741 & 0.015 & 0.904 & $0.915(0.214-3.906)$ \\
\hline
\end{tabular}

not significantly correlated with endocrine therapy, radiotherapy, targeted therapy, chemotherapy regimen, or chemotherapy cycle (all $\mathrm{P}>0.05$; Table 5).

\section{Discussion}

\section{Study findings}

Postoperative chemotherapy reduces the postoperative recurrence and mortality rates in patients with invasive breast cancer and has played an important role in the postoperative adjuvant treatment of invasive breast cancer. However, chemotherapy is associated with a variety of side effects. While the effects of chemotherapy on the gastrointestinal tract, skin, hair, and bone marrow have been widely investigated, few studies have been performed that evaluate its effects on glucose and lipid metabolism. In our current study, we explored the effects of postoperative chemotherapy on blood glucose and lipids in patients with invasive breast cancer and found postoperative chemotherapy could lead to the increased levels triglyceride and fasting blood glucose and decreased HDL in patients with breast cancer. Further multivariate logistic regression analysis showed that anthracycline-based chemotherapy was a protective factor for the increase of fasting blood glucose, whereas more than 6 cycles of chemotherapy was a risk factor for the increase of fasting blood glucose.

\section{Effect of postoperative chemotherapy on blood lipids in patients with invasive breast cancer}

The relevant mechanisms remain unclear. Although postoperative adjuvant chemotherapy can kill residual tumor cells, it can also damage some normal tissue cells, causing systemic oxidative stress and cellular catabolism, which may produce abnormal lipids. Changes in lifestyle (e.g., diet and exercise) during postoperative adjuvant chemotherapy for breast cancer may also affect patients' lipid metabolism. Anthracycline-based chemotherapy regimens have been shown to depress peroxisome proliferators, thus decreasing ATP-binding cassette transporter A1 (ABCA1) via an activation of the peroxisomal proliferator activated receptor $\gamma(\operatorname{PPAR} \gamma)$ and liver $\mathrm{X}$ receptor $\alpha(\mathrm{LXR} \alpha)$ transcription factors, which can result in blood lipid disorders (8). Other research has shown that neoadjuvant chemotherapy can cause a significant elevation of triglycerides, total cholesterol, and LDL in breast cancer patients, with anthracycline regimens having less impact on lipid profile 
compared to taxane-containing regimens (6). In our current study, the triglyceride levels significantly increased after chemotherapy $(1.81 \pm 1.02$ vs. $1.26 \pm 0.67 \mathrm{mmol} / \mathrm{L}$, $\mathrm{P}=0.000)$ whereas the levels of HDL significantly decreased $(1.11 \pm 0.29$ vs. $1.32 \pm 0.33 \mathrm{mmol} / \mathrm{L}, \mathrm{P}=0.000)$ in patients with invasive breast cancer. There were no significant changes in the levels of total cholesterol and LDL before and after chemotherapy. One study found that chemotherapy might also cause abnormalities in lipid metabolism in breast cancer patients, especially in younger patients (7). In another study, the incidence of dyslipidemia was shown to be low in breast cancer patients, and chemotherapy resulted in a significantly higher incidence of dyslipidemia; the authors thus recommended that lipid metabolism should be tested during chemotherapy (9). Dyslipidemia has been shown to be associated with the efficacy and sensitivity of chemotherapeutic treatment (10). In addition, dyslipidemia can trigger cardiovascular diseases in breast cancer patients (11-14). Therefore, blood lipids need to be closely monitored and managed in patients undergoing adjuvant chemotherapy for breast cancer.

\section{Effect of postoperative chemotherapy on fasting blood glucose in patients with invasive breast cancer}

Chemotherapy may lead to weight gain in breast cancer patients (15-18). Obesity not only leads to decreased quality of life and increased incidence of cardiovascular disease but also increases the risk of postoperative recurrence in breast cancer patients (19). Abnormalities in adipokines, insulin, and insulin-like growth factors have been suggested as potential contributors to weight gain. Adipokines, insulin, and insulin-like growth factors are all major factors affecting glucose metabolism, and therefore adjuvant chemotherapy can lead to abnormal glucose metabolism in breast cancer patients. A study performed in 2009 showed that neoadjuvant chemotherapy could lead to increased blood glucose in patients (20). Similarly, in our current study, the triglycerides were significantly increased after chemotherapy, and further multivariate logistic regression analysis showed that anthracycline-based chemotherapy was a protective factor for elevated fasting blood glucose [P $=0.035$, 95\% CI: 0.248 (0.068-0.908)], whereas more than 6 cycles of chemotherapy was a risk factor for the increase of fasting blood glucose [P=0.026, 95\% CI: 4.036 (1.17813.825)]. Anthracyclines (including adriamycin, epirubicin, erythromycin, and aclacinomycin) are cardiotoxic but have relatively small effects on blood glucose and lipid profiles.
In contrast, long-course chemotherapy has greater effects on adipokines, insulin, and insulin-like growth factors and thus increases the risk for abnormally elevated blood glucose.

\section{Limitation}

The number of patient samples in this study is too small, and a large sample study is needed to confirm these results. Moreover, due to the limitation of this retrospective study, we failed to study the different effects of other chemotherapy drugs on the glucose metabolism.

\section{Summary}

Anthracycline-based chemotherapy is a protective factor for the increase of fasting blood glucose, and more than 6 cycles of chemotherapy is a risk factor for the increase of fasting blood glucose.

\section{Conclusions}

Postoperative chemotherapy can lead to the elevated triglyceride and fasting blood glucose and decreased HDL in patients with breast cancer. Anthracycline-based chemotherapy is a protective factor for the increase of fasting blood glucose, and more than 6 cycles of chemotherapy is a risk factor for the increase of fasting blood glucose.

\section{Acknowledgments}

Funding: None.

\section{Footnote}

Reporting Checklist: The authors have completed the STROBE reporting checklist. Available at http://dx.doi. org/10.21037/gs-21-141

Data Sharing Statement: Available at http://dx.doi. org/10.21037/gs-21-141

Conflicts of Interest: All authors have completed the ICMJE uniform disclosure form (available at http://dx.doi. org/10.21037/gs-21-141). The authors have no conflicts of interest to declare.

Ethical Statement: The authors are accountable for all 
aspects of the work in ensuring that questions related to the accuracy or integrity of any part of the work are appropriately investigated and resolved. The study protocol was consistent with the Declaration of Helsinki (as revised in 2013. The approval from ethics board was not required since our study was a retrospectively observational study, and we only studied the clinical data of the patients, which would not bring any harm to the patients. Individual consent for this retrospective analysis was waived.

Open Access Statement: This is an Open Access article distributed in accordance with the Creative Commons Attribution-NonCommercial-NoDerivs 4.0 International License (CC BY-NC-ND 4.0), which permits the noncommercial replication and distribution of the article with the strict proviso that no changes or edits are made and the original work is properly cited (including links to both the formal publication through the relevant DOI and the license). See: https://creativecommons.org/ licenses/by-nc-nd/4.0/.

\section{References}

1. Youlden DR, Baade PD, Walker R, et al. Breast Cancer Incidence and Survival Among Young Females in Queensland, Australia. J Adolesc Young Adult Oncol 2020;9:402-9.

2. Katalinic A, Eisemann N, Kraywinkel K, et al. Breast cancer incidence and mortality before and after implementation of the German mammography screening program. Int J Cancer 2020;147:709-18.

3. Lagacé F, Ghazawi FM, Le M, et al. Analysis of incidence, mortality trends, and geographic distribution of breast cancer patients in Canada. Breast Cancer Res Treat 2019;178:683-91.

4. Azamjah N, Soltan-Zadeh Y, Zayeri F. Global Trend of Breast Cancer Mortality Rate: A 25-Year Study. Asian Pac J Cancer Prev 2019;20:2015-20.

5. Mejdahl MK, Wohlfahrt J, Holm M, et al. Breast cancer mortality in synchronous bilateral breast cancer patients. Br J Cancer 2019;120:761-7.

6. He T, Wang C, Tan Q, et al. Adjuvant chemotherapyassociated lipid changes in breast cancer patients: A real-word retrospective analysis. Medicine (Baltimore) 2020;99:e21498.

7. Tian W, Yao Y, Fan G, et al. Changes in lipid profiles during and after (neo)adjuvant chemotherapy in women with early-stage breast cancer: A retrospective study. PLoS
One 2019; 14:e0221866.

8. Sharma M, Tuaine J, McLaren B, et al. Chemotherapy Agents Alter Plasma Lipids in Breast Cancer Patients and Show Differential Effects on Lipid Metabolism Genes in Liver Cells. PLoS One 2016;11:e0148049.

9. Li X, Liu ZL, Wu YT, et al. Status of lipid and lipoprotein in female breast cancer patients at initial diagnosis and during chemotherapy. Lipids Health Dis 2018;17:91.

10. Qu F, Chen R, Peng Y, et al. Assessment of the Predictive Role of Serum Lipid Profiles in Breast Cancer Patients Receiving Neoadjuvant Chemotherapy. J Breast Cancer 2020;23:246-58.

11. Kim AS, Conte MS. Specialized pro-resolving lipid mediators in cardiovascular disease, diagnosis, and therapy. Adv Drug Deliv Rev 2020;159:170-9.

12. Davidson J, Rotondo D. The potential role of the lipid phosphate phosphatase 3 (Plpp3) gene in cardiovascular disease. Curr Opin Lipidol 2020;31:258-9.

13. Mourikis P, Zako S, Dannenberg L, et al. Lipid lowering therapy in cardiovascular disease: From myth to molecular reality. Pharmacol Ther 2020;213:107592.

14. De Luca L, Arca M, Temporelli PL, et al. Current lipid lowering treatment and attainment of LDL targets recommended by ESC/EAS guidelines in very high-risk patients with established atherosclerotic cardiovascular disease: Insights from the START registry. Int J Cardiol 2020;316:229-35.

15. Fadelu T, Damuse R, Lormil J, et al. Body Mass Index, Chemotherapy-Related Weight Changes, and DiseaseFree Survival in Haitian Women With Nonmetastatic Breast Cancer. JCO Glob Oncol 2020;6:1656-65.

16. Jung GH, Kim JH, Chung MS. Changes in weight, body composition, and physical activity among patients with breast cancer under adjuvant chemotherapy. Eur J Oncol Nurs 2020;44:101680.

17. de Kruif JTCM, Visser M, van den Berg MMGA, et al. A longitudinal mixed methods study on changes in body weight, body composition, and lifestyle in breast cancer patients during chemotherapy and in a comparison group of women without cancer: study protocol. BMC Cancer 2019;19:7.

18. Morrison VA, McCall L, Muss HB, et al. The impact of actual body weight-based chemotherapy dosing and body size on adverse events and outcome in older patients with breast cancer: Results from Cancer and Leukemia Group B (CALGB) trial 49907 (Alliance A151436). J Geriatr Oncol 2018;9:228-34. 
19. Donegan WL, Hartz AJ, Rimm AA. The association of body weight with recurrent cancer of the breast. Cancer 1978, 41:1590-4.

20. Hickish T, Astras G, Thomas P, et al. Glucose intolerance

Cite this article as: Qi A, Li Y, Yan S, Sun H, Zhao M, Chen Y. Effect of postoperative chemotherapy on blood glucose and lipid metabolism in patients with invasive breast cancer. Gland Surg 2021;10(4):1470-1477. doi: 10.21037/gs-21-141 during adjuvant chemotherapy for breast cancer. J Natl Cancer Inst 2009;101:537.

(English Language Editor: J. Gray) 